\title{
Tendinopatía rotuliana. Modelo de actuación terapéutico en el deporte
}

\section{Patellar tendinopathy. Therapeutic model in the sport}

\section{Introducción}

El dolor tendinoso, conocido como tendinopatía, es muy común en individuos físicamente activos, ya sea a nivel competitivo como recreacional. Sin embargo, se ha demostrado que individuos físicamente inactivos también lo sufren. Por lo tanto, se puede afirmar que la actividad física no se puede asociar directamente a la histopatología, y que el ejercicio físico puede ser más importante en la provocación de los síntomas que en ser el causante de la lesión ${ }^{1,2}$. La sobreutilización induce esta condición, pero la etiología y la patogenia no están científicamente clarificadas.

En el tendón rotuliano se considera que el aparato extensor de la rodilla se ve sometido a determinados traumatismos repetidos, repentinos y balísticos, con momentos de fuerza muy elevados. En individuos en edad de crecimiento las tendinopatías de este tendón se presentan en puntos de osificación secundaria, bien en la tuberosidad anterior de la tibia (enfermedad de Osgood-Schlatter), bien en el polo inferior de la rótula (Sinding-Larsen-Johansson) ${ }^{3}$.

\section{Etiopatogenia de la tendinopatía rotuliana}

En series de estudios de la UEFA desde la temporada 2001-2002 a la 2008-2009 se contabilizaron 32 lesiones tendinosas en el total de equipos participantes en competiciones europeas, lo que representa un $6 \%$ del total de las lesiones. De estas 32 lesiones, 6 se presentaron en el tendón rotuliano (2,2\% del total $)^{4}$.

La teoría más aceptada es la del agotamiento por sobreuso, que conlleva un aumento de la rigidez muscular con una disminución de la extensibilidad del complejo musculotendinoso, y una menor

\footnotetext{
* Autor para correspondencia.

Correo electrónico: ricard.pruna@fcbarcelona.cat (R. Pruna).
}

capacidad de contracción rápida que hace que aumente la tracción sobre el tendón ${ }^{5,6}$.

El control de los factores de riesgo es indispensable en cualquier tipo de lesión. En el tendón rotuliano existen una serie de factores intrínsecos y extrínsecos que pueden contribuir de manera clara al desarrollo de esta dolencia (tablas 1 y 2). Diversos autores vinculan la tendinopatía rotuliana con disminuciones musculares del cuádriceps, condropatías rotulianas e hipermovilidad de la rótula. A pesar de esto, el principal factor etiológico se considera que es la desaceleración en el momento del aterrizaje en el suelo tras un salto $^{7}$. Lo que parece evidente es que existe una clara relación entre los desajustes biomecánicos de la rótula y las tendinopatías de inserción rotuliana.

\section{Diagnóstico}

La clasificación clínica más utilizada para la valoración de esta lesión es la escala de Blazina (1973) (tabla 3), que se basa en criterios de evolución del dolor según la funcionalidad. Igualmente, la escala de Victorian Institute of Sport Assessment (VISA-P) permite una clasificación clínica basada en la gravedad sintomática, la capacidad funcional y la capacidad deportiva ${ }^{8}$. Recientemente esta escala ha sido traducida y validada al español $\left(\right.$ VISA-P-Sp) ${ }^{9}$ (fig. 1).

La exploración clínica implica la valoración de posibles alineaciones incorrectas del aparato extensor, del ángulo $Q$ de Insall, de la pronación del retropié, de la asimetría de las extremidades inferiores, de la atrofia del cuádriceps y de la flexibilidad muscular de los isquiosurales, gastrocnemius $\mathrm{y}$ cuádriceps.

En el protocolo propuesto se valora el dolor en el tendón rotuliano y su implicación funcional mediante la escala de Blazina $^{10}$ (tabla 3 ), y por medio de la escala VISA-P-Sp para la tendinopatía rotuliana (Anexo 1 ).

0025-7753/\$ - see front matter ๑ 2012 Elsevier España, S.L. Todos los derechos reservados. http://dx.doi.org/10.1016/j.medcli.2012.12.006 
Tabla 1

Resumen de los factores intrínsecos asociados a tendinopatía por sobreuso (Paavola 2005)

\begin{tabular}{ll}
\hline Generales & Locales \\
\hline Sexo & Malalineaciones: pie hiperpronado \\
& o hipopronado, genu valgo/varo, \\
& anteversión del cuello femoral, etc. \\
Edad & Dismetría de miembros inferiores \\
& Debilidades musculares \\
& Desequilibrios musculares \\
& Laxitud articular \\
& Disminución de la flexibilidad \\
\hline
\end{tabular}

El diagnóstico por imagen se realiza por ecografía, ya que aporta una buena imagen del estado de las fibras de colágeno, así como de la posible existencia de neovascularización alrededor del tendón (fig. 2). Una de las claves de la ecografía es que se trata de una exploración dinámica que permite observar la funcionalidad del tendón, así como complementar la exploración clínica. De igual manera, es una prueba que en el ámbito de la medicina deportiva es de disponibilidad prácticamente inmediata.

La resonancia magnética (RM) aporta datos sobre el estado de otras estructuras articulares y es vital en el diagnóstico diferencial. Una de las ventajas de la RM frente a la ecografía es que proporciona una visión reproducible del área de estudio en múltiples planos y permite descartar otras enfermedades de origen no tendinoso ${ }^{11}$ (fig. 3).

\section{Tratamiento}

Tanto en la fase aguda como en la crónica, el tratamiento será igual exceptuando las pautas de ejercicios excéntricos. Se aplicará crioterapia durante unos $15-20$ min 3-4 veces al día y se seguirá una secuencia que integre la bicicleta o trabajo sin impacto para realizar el calentamiento del tendón. Es posible realizar el trabajo al $60-70 \%$ del peso corporal a baja intensidad en tapiz rodante antigravedad. En este protocolo de actuación existen distintas opciones terapéuticas que se barajan individualizando cada caso y que se exponen a continuación.

\section{Electroterapia}

La electroterapia es cualquier forma de tratamiento en la que la fuente eléctrica constituye parte principal del agente físico, por lo que también se incluyen la obtención de radiación electromagnética (corrientes de alta frecuencia) y otras formas de energía, como los ultrasonidos, el láser o la magnetoterapia. Desde un punto de vista científico, no existe evidencia del tipo más adecuado para el tratamiento de la patología tendinosa ${ }^{12,13}$.

En los últimos años ha cobrado interés la denominada electrólisis percutánea intratendinosa, que consiste en la aplicación de corrientes galvánicas intratendinosas. Sin embargo, no

Tabla 2

Resumen de factores extrínsecos (Paavola 2005, Brukner 2007)

\footnotetext{
Métodos de entrenamiento

Duración o intensidad excesiva

Déficit de adaptación fisiológica

Inadaptación a la especificidad del entrenamiento

Incrementos súbitos en el programa de entrenamiento

Errores en la adaptación individual al entrenamiento

Cambios de superficie de entrenamiento/juego

Calentamiento insuficiente

Entrenamiento general inadecuado

Recuperación insuficiente

Problemas derivados del material
}

Tabla 3

Clasificación de Blazina (1973)

Estadio 1: el dolor solo aparece después de la actividad deportiva y no influye en el rendimiento del deportista

Estadio 2: el paciente presenta dolor en el inicio de la actividad deportiva, pero con el calentamiento desaparece y tampoco afecta de forma significativa al rendimiento deportivo

Estadio 3: el dolor está presente durante y después de la actividad deportiva, provocando incluso que el deportista se vea obligado a dejar su actividad deportiva

hemos podido encontrar en la literatura médica ninguna investigación básica y/o clínica que justifique el uso de este tipo de corriente en el tratamiento de la enfermedad tendinosa. Actualmente, esta técnica debe considerarse experimental.

\section{Terapia manual}

Consiste en masaje de descarga del cuádriceps crural con movilización de la rótula y un calentamiento del tendón preactividad deportiva o sesión de tratamiento. Se realiza, de igual manera, una extensibilidad manual del tendón transversal, y longitudinal para los casos en que hay afectación del paratendón, y estiramientos en tensión activa de cuádriceps aplicándolos también en la cadena posterior.

\section{Ejercicios excéntricos}

El punto clave en el tratamiento de las tendinopatías rotulianas se basa en un protocolo de ejercicios excéntricos. Son diversos los estudios que avalan este tipo de protocolos ${ }^{14,15}$.

El trabajo excéntrico provoca una mayor hipertrofia que el trabajo convencional de fuerza concéntrica o isométrica ${ }^{16-18}$, hecho de vital importancia para el cuádriceps en estas lesiones.

La pauta de ejercicios en la fase aguda se realiza durante 12 semanas seguidas. En caso de que el deportista esté en la fase final de la recuperación, se mantendrá 12 semanas a partir de ese momento. Los ejercicios se prescriben 2 veces al día los 7 días de la semana mediante 6 series de 10 repeticiones, realizando la bajada (fase excéntrica) sobre la pierna afectada o 2 piernas y la subida (fase concéntrica) sobre la pierna sana o 2 piernas.

El trabajo excéntrico manual consiste en 4 series de 12 repeticiones, alternando la velocidad de ejecución. El deportista se sentará en el borde de la camilla, manteniendo una contracción isométrica, y será el terapeuta, con los brazos, quien haga la resistencia para generar una contracción excéntrica. Hay que respetar en esta fase el dolor del deportista. A medida que se toleren los ejercicios excéntricos de forma manual se podrá comenzar con el resto de ejercicios excéntricos.

En cuanto a la fase crónica, las primeras sesiones enfatizarán en los ejercicios que se explican en el protocolo de trabajo de las tendinopatías rotulianas en fase aguda. La progresión al siguiente grupo de ejercicios (opciones A, B, C y D del Anexo 1, disponible en la versión on line) se realiza una vez se han superado satisfactoriamente los de la fase aguda. En primer lugar se realizarán los ejercicios A y B, y posteriormente, antes de la fase de readaptación e incorporación a los entrenamientos, los ejercicios C y D.

En caso de que el dolor no exista se puede aumentar la carga añadiendo una mochila con pesos ${ }^{19,20}$.

\section{Factores de crecimiento autólogos (plasma rico en plaquetas)}

El plasma rico en plaquetas (PRP) se define como una muestra de sangre autóloga con una alta concentración de plaquetas. Estas contienen gránulos que secretan diferentes tipos de factores de 
Cuestionario de valoración VISA-P (Victorian Institute of Sports Assessment) : TENDINOPATÍA ROTULIANA

Este es un cuestionario para la valoración de la gravedad de los síntomas en individuos con tendinopatía rotuliana. El término "dolor" en el cuestionario hace referencia a la zona específica del tendón rotuliano. Para indicar su intensidad de dolor, por favor, marque de 0 a 10 en la escala teniendo en cuenta que $0=$ ausencia de dolor y 10 = máximo dolor que imagina.

1.- ¿Durante cuántos minutos puede estar sentado sin dolor?

\begin{tabular}{c|c|c|c|c|c|}
\hline $\mathbf{0 - 1 5} \mathbf{~ m i n}$ & $\mathbf{1 5 - 3 0} \mathbf{~ m i n}$ & $\mathbf{3 0 - 6 0} \mathbf{~ m i n}$ & $\mathbf{6 0 - 9 0} \mathbf{~ m i n}$ & $\mathbf{9 0 - 1 2 0} \mathbf{~ m i n}$ & $\mathbf{7 1 2 0} \mathbf{~ m i n}$ \\
\hline 0 & 2 & 4 & 6 & 8 & 10
\end{tabular}

2.- ¿Le duele al bajar escaleras con paso normal?

Sin dolor

\begin{tabular}{|l|l|l|l|l|l|l|l|l|l|l|}
\hline $\mathbf{0}$ & $\mathbf{1}$ & $\mathbf{2}$ & $\mathbf{3}$ & $\mathbf{4}$ & $\mathbf{5}$ & $\mathbf{6}$ & $\mathbf{7}$ & $\mathbf{8}$ & $\mathbf{9}$ & $\mathbf{1 0}$ \\
\hline 10 & 9 & 8 & 7 & 6 & 5 & 4 & 3 & 2 & 1 & 0
\end{tabular}

Dolor muy intenso

3.- ¿Le duele la rodilla al extenderla completamente sin apoyar el pie en el suelo?

Sin dolor

\begin{tabular}{|c|c|c|c|c|c|c|c|c|c|c|}
\hline $\mathbf{0}$ & $\mathbf{1}$ & $\mathbf{2}$ & $\mathbf{3}$ & $\mathbf{4}$ & $\mathbf{5}$ & $\mathbf{6}$ & $\mathbf{7}$ & $\mathbf{8}$ & $\mathbf{9}$ & $\mathbf{1 0}$ \\
\hline 10 & 9 & 8 & 7 & 6 & 5 & 4 & 3 & 2 & 1 & 0 \\
\hline
\end{tabular}

Dolor muy intenso 4.- ¿Tiene dolor en la rodilla al realizar un gesto de "zancada"
(flexión de rodilla tras un movimiento amplio hacia delante con carga
completa del peso corporal sobre la pierna adelantada)

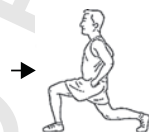

Sin dolor

\begin{tabular}{|c|c|c|c|c|c|c|c|c|c|c|}
\hline $\mathbf{0}$ & $\mathbf{1}$ & $\mathbf{2}$ & $\mathbf{3}$ & $\mathbf{4}$ & $\mathbf{5}$ & $\mathbf{6}$ & $\mathbf{7}$ & $\mathbf{8}$ & $\mathbf{9}$ & $\mathbf{1 0}$ \\
\hline 10 & 9 & 8 & 7 & 6 & 5 & 4 & 3 & 2 & 1 & 0
\end{tabular}

Dolor muy intenso
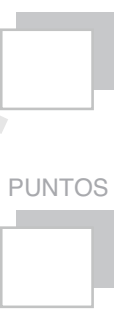

\section{5.- ¿Tiene problemas para ponerse en cuclillas?}

Sin problemas

\begin{tabular}{|l|l|l|l|}
\hline $\mathbf{0}$ & $\mathbf{1}$ & $\mathbf{2}$ & $\mathbf{3}$ \\
\hline 10 & 9 & 8
\end{tabular}

6.- ¿Le duele al hacer 10 saltos seguidos sobre la pierna afectada o Inmediatamente después de hacerlos?

Sin dolor

\begin{tabular}{|l|l|l|l|l|l|l|l|l|l|l|}
\hline $\mathbf{0}$ & $\mathbf{1}$ & $\mathbf{2}$ & $\mathbf{3}$ & $\mathbf{4}$ & $\mathbf{5}$ & $\mathbf{6}$ & $\mathbf{7}$ & $\mathbf{8}$ & $\mathbf{9}$ & $\mathbf{1 0}$ \\
\hline 10 & 9 & 8 & 7 & 6 & 5 & 4 & 3 & 2 & 1 & 0
\end{tabular}

Incapaz

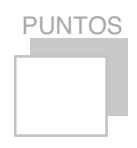

PUNTOS

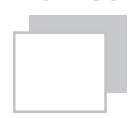

PUNTOS

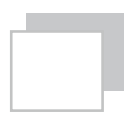

crecimiento, los cuales desempeñan un papel muy importante en el proceso de cicatrización normal. Estudios realizados en células de cultivo han demostrado que el PRP puede estimular el proceso asociado a la reparación tendinosa. Partiendo de esta base, se administran inyecciones de PRP a los pacientes con tendinopatía para promover la reparación tendinosa ${ }^{21}$. Sin embargo, la mayoría de los estudios realizados in vivo muestran resultados contradictorios $^{22,23}$. Tal y como recomienda el Comité Olímpico Internacional en su documento de consenso, es necesario llevar a cabo más investigación básica y clínica antes de poder recomendar el uso de PRP en tendones lesionados. Para cada atleta en particular hay que evaluar el riesgo/beneficio antes de recomendar esta modalidad terapéutica, actualmente no validada de forma científica ${ }^{24-26}$.

Dolor muy

intenso/ Incapaz
Ondas de choque

Las ondas de choque son una opción terapéutica a tener en cuenta en caso de fracaso de las medidas de tratamiento conservador convencionales en la tendinopatía rotuliana $\mathrm{cr}$ ónica, dado que se han documentado mejorías en cuanto al dolor y a la funcionalidad ${ }^{27-29}$ y ningún efecto adverso serio ha sido descrito $^{30}$. Recomendamos un tratamiento con ondas de choque de baja energía a días alternos $(2.000$ pulsos a $15 \mathrm{~Hz}$ 

7.- ¿Practica algún deporte o actividad física en la actualidad?
PUNTOS
$0 \square$ No, en absoluto
$4 \square$ Entrenamiento modificado y/o competición modificada
$7 \square$ Entrenamiento completo y/o competición, pero a menor nivel que cuando empezaron los síntomas
$10 \square$ Competición al mismo nivel o mayor que cuando empezaron los síntomas

8.- Por favor, conteste A, B o C en esta pregunta según el estado actual de su lesión:

- Si no tiene dolor al realizar deporte, por favor, conteste sólo a la pregunta 8A

- Si tiene dolor mientras realiza el deporte pero éste no le impide completar la actividad, por favor, conteste únicamente la pregunta 8B

- Si tiene dolor en la rodilla y éste le impide realizar deporte, por favor, conteste solamente la pregunta 8C

8A.- Si no tiene dolor mientras realiza deporte, ¿cuánto tiempo puede estar entrenando o practicando?

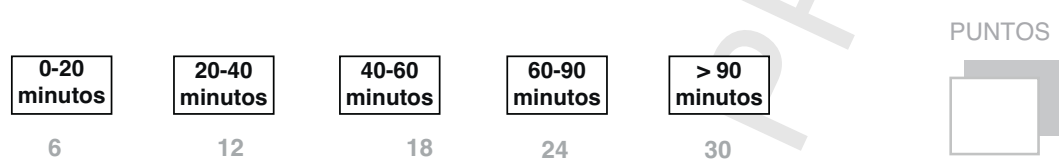

8B.- Si tiene cierto dolor mientras realiza deporte pero éste no obliga a interrumpir el entrenamiento o la actividad física, ¿cuánto tiempo puede estar entrenando o haciendo deporte?

\begin{tabular}{|c|c|c|c|c|c|}
\hline $\begin{array}{c}0-15 \\
\text { minutos }\end{array}$ & $\begin{array}{c}15-30 \\
\text { minutos }\end{array}$ & $\begin{array}{c}\text { 30-45 } \\
\text { minutos }\end{array}$ & $\begin{array}{c}45-60 \\
\text { minutos }\end{array}$ & \begin{tabular}{c|}
$>60$ \\
minutos
\end{tabular} & \\
\hline 0 & 5 & 10 & 15 & 20 & \\
\hline
\end{tabular}

8C.- Si tiene dolor que le obliga a detener el entrenamiento o práctica deportiva, ¿cuánto tiempo puede aguantar haciendo el deporte o la actividad física?

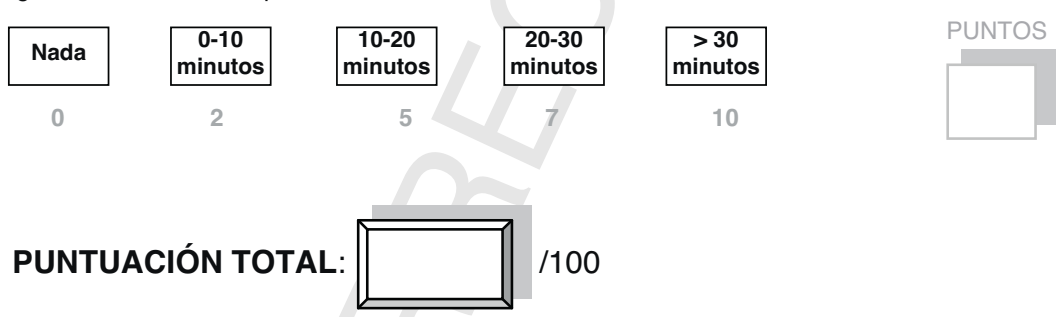

Nombre: Fecha: perilesionales seguidos de 1.000 pulsos a $7-8 \mathrm{~Hz}$ focalizados en la zona exacta del dolor), combinada con un programa de ejercicios excéntricos.

\section{Inyección con grandes volúmenes}

Cuando otros tratamientos conservadores no han funcionado, la inyección de grandes volúmenes de suero fisiológico (en torno a $40 \mathrm{ml}$ ) como tratamiento en la tendinopatía rotuliana crónica incapacitante $\mathrm{y}$ dolorosa busca romper mediante efectos mecánicos los neovasos (eco-doppler positivo) que penetran por la cara profunda del tendón y la grasa de Hoffa. A las $72 \mathrm{~h}$ se debe comenzar un programa de ejercicios exc éntricos ${ }^{31}$.

\section{Retorno a la competición}

Como es conocido en todo tipo de lesiones, la decisión del retorno a la competición es sumamente compleja e implica la sincronización de determinados requisitos. En el caso de la tendinopatía rotuliana se propone basarse primeramente en la escala VISA-P-Sp. En función de la puntuación obtenida se aplicarán los siguientes criterios: no podrán volver al trabajo de campo los deportistas que estén por debajo de una puntuación de 50; para incorporarlos al grupo deberán tener una puntuación por encima de $60 \mathrm{y}$, finalmente, deberán haber mejorado en al menos 30 puntos desde la primera vez que se pasó el cuestionario, siempre y cuando se sobrepasen los 60 puntos. 


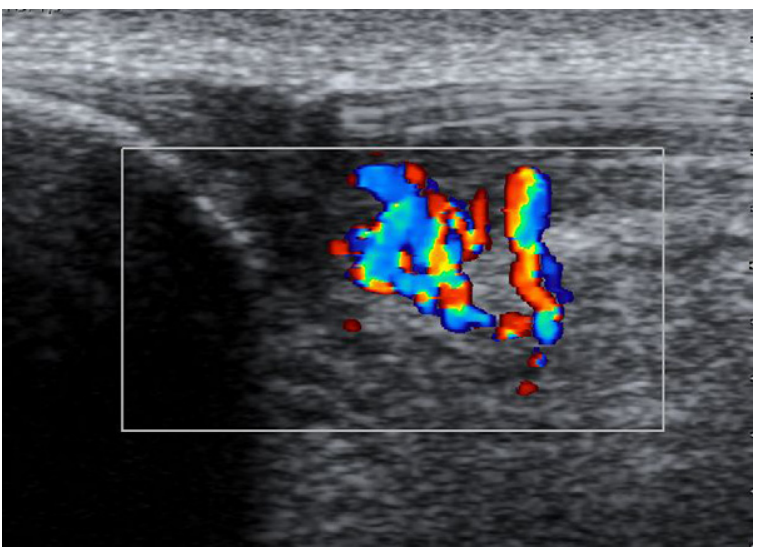

Figura 2. Imagen ecográfica de formación de neovasos en la degeneración del tendón.

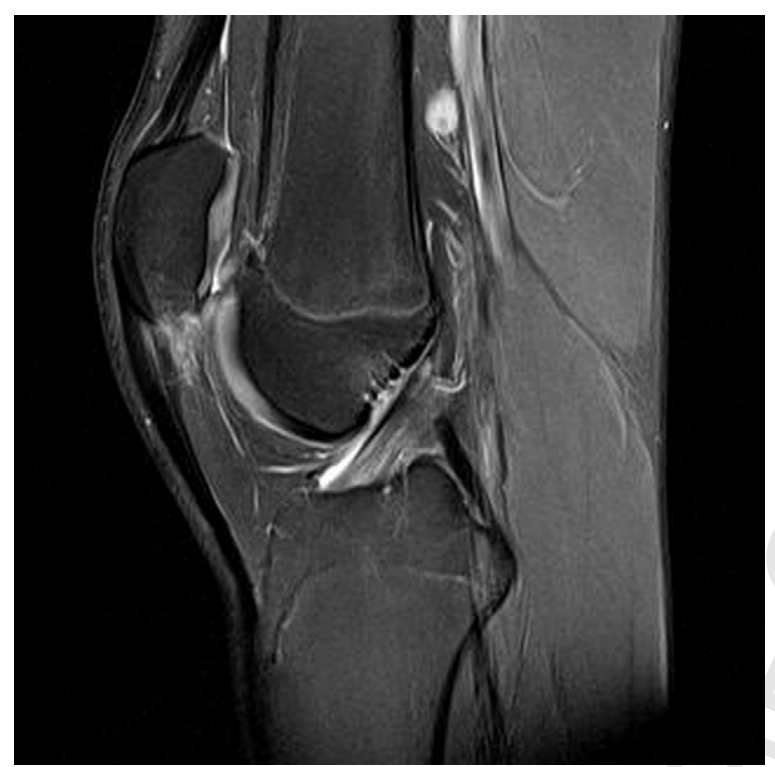

Figura 3. Imagen por resonancia magnética de una degeneración del tendón rotuliano.

Como parte del proceso de decisión del retorno a la competición se debe tener en cuenta la sensación subjetiva de dolor al hacer los ejercicios excéntricos A, B, C y D de los propuestos con anterioridad. Este dolor se debe haber reducido a la mitad con respecto al del inicio del tratamiento, valorado con escala visual analógica (puntuación de 0 a 10).

Desde el punto de vista médico es básico tener en consideración que el deportista debe haber completado al menos 5 sesiones completas de entrenamiento con el grupo y debe poder realizar sin molestia alguna ejercicios con impacto y saltos realizados individualmente. La recuperación de los niveles de fuerza y de los volúmenes de los diferentes grupos musculares debe ser simétrica a los del lado contralateral y, finalmente, en la imagen ecográfica debe haber desaparecido la neovascularización alrededor del tendón.

\section{Anexo. Material adicional}

Se puede consultar material adicional a este artículo en su versión electrónica disponible en http://dx.doi.org/10.1016/j.medcli.2012.12.006.

\section{Referencia no citada}

32.

\section{Bibliografía}

1. Alfredson $\mathrm{H}$. The chronic painful Achilles and patellar tendon: Research on basic biology and treatment. Scand J Med Sci Sports. 2005;15:252-9.

2. Young MA, Cook JL, Purdam CR, Kiss ZS, Alfredson H. Eccentric decline squat protocol offers superior results at 12 months compared with traditional eccentric protocol for patellar tendinopathy in volleyball players. Br J Sports Med. 2005;39:102-5.

3. Medlar RC, Lyne ED. Sinding-Larsen-Johansson disease. Its etiology and natural history. J Bone Joint Surg Am. 1978;60:1113-6.

4. Ekstrand J, Hägglund $M$, Waldén $M$. Injury incidence and injury patterns in professional football: the UEFA injury study. Br J Sports Med. 2011;45:553-8.

5. Basso O, Amis AA, Race A, Johnson DP. Patellar tendon fiber strains: Their differential responses to quadriceps tension. Clin Orthop Relat Res. 2002;246-53.

6. Micheli LJ, Fehlandt Jr AF. Overuse injuries to tendons and apophyses in children and adolescents. Clin Sports Med. 1992;11:713-26.

7. Richards DP, Ajemian SV, Wiley JP, Zernicke RF. Knee joint dynamics predict patellar tendinitis in elite volleyball players. Am J Sports Med. 1996;24:676-83.

8. Robinson JM, Cook JL, Purdam C, Visentini PJ, Ross J, Maffulli N, et al. The VISA-A questionnaire: A valid and reliable index of the clinical severity of Achilles tendinopathy. Br J Sports Med. 2001;35:335-41.

9. Hernandez-Sanchez S, Hidalgo MD, Gomez A. Cross-cultural adaptation of VISAP score for patellar tendinopathy in Spanish population. J Orthop Sports Phys Ther. 2011;41:581-91.

10. Blazina ME, Kerlan RK, Jobe FW, Carter VS, Carlson GJ. Jumper's knee. Orthop Clin North Am. 1973;4:665-78.

11. Hughes TH. Imaging of tendon ailments. En: Maffulli N, Renström P, Leadbetter $\mathrm{W}$, editors. Tendon injuries: Basic science and clinical medicine. London: Springer-Verlag London Limited; 2005.

12. Leadbetter J. The effect of therapeutic modalities on tendinopathy. En: Maffulli N, Renström P, Leadbetter W, editors. Tendon injuries: Basic science and clinical medicine. London: Springer-Verlag London Limited; 2005

13. Andrade Ortega JA. Electroterapia. En: Sánchez I, Ferrero A, Aguilar JJ, et al. editors. Manual SERMEF de Rehabilitación y Medicina Física. Buenos Aires; Madrid: Medicina Panamericana; 2006.

14. Alfredson $\mathrm{H}$, Pietila T, Jonsson P, Lorentzon R. Heavy-load eccentric calf muscle training for the treatment of chronic Achilles tendinosis. Am J Sports Med. 1998;26:360-6.

15. Curwin S, Stanish W. Tendinitis: It's etiology and treatment. 1st ed, Lexington: Collamore Press; 1984.

16. Bosco C, Cardinale M, Tsarpela O. Influence of vibration on mechanical power and electromyogram activity in human arm flexor muscles. Eur J Appl Physiol Occup Physiol. 1999;79:306-11.

17. Issurin VB. Vibrations and their applications in sport. A review. J Sports Med Phys Fitness. 2005;45:324-36.

18. Issurin VB, Tenenbaum G. Acute and residual effects of vibratory stimulation on explosive strength in elite and amateur athletes. J Sports Sci. 1999;17:177-82.

19. Clarkson PM, Nosaka K, Braun B. Muscle function after exercise-induced muscle damage and rapid adaptation. Med Sci Sports Exerc. 1992;24:512-20.

20. Donnelly AE, Clarkson PM, Maughan RJ. Exercise-induced muscle damage: Effects of light exercise on damaged muscle. Eur J Appl Physiol Occup Physiol. 1992;64:350-3.

21. Hoksrud AF, Bahr R. Injectable agents derived from or targeting vascularity: Has clinical acceptance in managing tendon disorders superseded scientific evidence? J Musculoskelet Neuronal Interact. 2011;11:174-84.

22. De Almeida AM, Demange MK, Sobrado MF, Rodrigues MB, Pedrinelli A, Hernandez AJ. Patellar tendon healing with platelet-rich plasma: A prospective randomized controlled trial. Am J Sports Med. 2012;40:1282-8.

23. Owens Jr RF, Ginnetti J, Conti SF, Latona C. Clinical and magnetic resonance imaging outcomes following platelet rich plasma injection for chronic midsubstance Achilles tendinopathy. Foot Ankle Int. 2011;32:1032-9.

24. Maffulli N, del Buono A. Platelet plasma rich products in musculoskeletal medicine: Any evidence? Surgeon. 2012;10:148-50.

25. Del Buono A, Papalia R, Denaro V, Maccauro G, Maffulli N. Platelet rich plasma and tendinopathy: State of the art. Int J Immunopathol Pharmacol. 2011;24(1 Suppl 2):79-83.

26. Paoloni J, de Vos RJ, Hamilton B, Murrell GA, Orchard J. Platelet-rich plasma treatment for ligament and tendon injuries. Clin J Sport Med. 2011:21:37-45.

27. Wang CJ, Ko JY, Chan YS, Weng LH, Hsu SL. Extracorporeal shockwave for chronic patellar tendinopathy. Am J Sports Med. 2007;35:972-8.

28. Vulpiani MC, Vetrano M, Savoia V, di Pangrazio E, Trischitta A, Ferretti A. Jumper's knee treatment with extracorporeal shock wave therapy: A long-term follow-up observational study. J Sports Med Phys Fitness. 2007;47:323-8.
223

224

225

226

227

228

229

230

231

4232

233

5234

235

236

237

238

239

240

241

242

243

244

245

246

247

248

249

250

251

252

253

254
255

256

257

258

259

260

261

262

263

264

265

266

267

268

269

270

271

272

273

274

275

276
277

Q7278

279

280

281

282

283
284

285

286

287

288

289

290

291

292

293

Cómo citar este artículo: Pruna R, et al. Tendinopatía rotuliana. Modelo de actuación terapéutico en el deporte. Med Clin (Barc). 2013. http://dx.doi.org/10.1016/j.medcli.2012.12.006 
29. Van der Worp H, Zwerver J, van den Akker-Scheek I, Diercks RL. The TOPSHOCK study: Effectiveness of radial shockwave therapy compared to focused shockwave therapy for treating patellar tendinopath - design of a randomised controlled trial. BMC Musculoskelet Disord. 2011;12:229.

30. Van Leeuwen MT, Zwerver J, van den Akker-Scheek I. Extracorporeal shockwave therapy for patellar tendinopathy: A review of the literature. Br J Sports Med. 2009;43:163-8.
31. Crisp T, Khan F, Padhiar N, Morrisey D, King J, Jalan R, et al. High volume ultrasound guided injections at the interface between the patellar tendon and Hoffa's body are effective in chronic patellar tendinopathy: A pilot study. Q8 Disabil Rehabil. 2008;30:1625-34.

32. Fredberg U, Bolvig L, Andersen NT. Prophylactic training in asymptomatic soccer players with ultrasonographic abnormalities in Achilles and patellar tendons: The Danish Super League Study. Am J Sports Med. 2008;36:451-60.

Cómo citar este artículo: Pruna R, et al. Tendinopatía rotuliana. Modelo de actuación terapéutico en el deporte. Med Clin (Barc). 2013. http://dx.doi.org/10.1016/j.medcli.2012.12.006 